Canadian Oncology

Nursing Journal

Revue canadienne

de soins infirmiers

en oncologie

Volume 28, Issue 3 • Summer 2018

elSSN: 2368-8076 


\title{
Approches favorisant le sentiment de proximité chez le patient dans un centre de cancérologie ambulatoire
}

\author{
par Chloe Grover, Erin Mackasey, Erin Cook, Lucie Tremblay et Carmen G. Loiselle
}

\section{RÉSUMÉ}

But: Cette étude a permis d'explorer le sentiment de proximité chez les patients d'un service de chimiothérapie ambulatoire.

Méthodologie : À l'aide d'un devis qualitatif descriptif, 10 participants présentant divers cancers ont été recrutés parmi les patients d'un grand centre de cancérologie de Montréal, au Québec. Les entrevues individuelles, enregistrées sur cassette, ont été transcrites textuellement. Ces verbatim ont ensuite été codés et analysés pour être catégorisés par thèmes.

Résultats : En ce qui a trait à l'approche de l'équipe soignante, les participants ont exprimé leur besoin d'être considérés avant tout pour la personne qu'ils sont, ensuite comme un patient à traiter. Ils ont en outre fait ressortir limportance de : 1) se sentir bien accueillis dans le milieu des soins aux personnes atteintes de cancer; 2) recevoir des soins adaptés à la personne et au contexte; et 3) tenir compte de leurs rôles professionnel et social, qui vont au-delà de leur rôle de «malade».

La communication réciproque d'informations patient-infirmière augmenterait également le sentiment de bénéficier d'une approche personnalisée.

\section{AU SUJET DES AUTEURES}

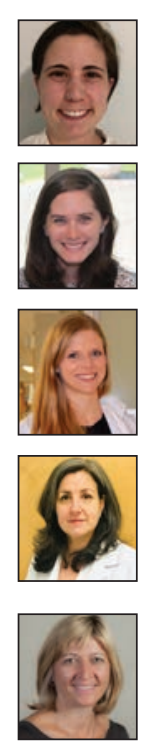

Chloe Grover, inf. aut., M.Sc., École de sciences infirmières

Ingram, Université McGill, Montréal, QC

Erin Mackasey, inf. aut., M.Sc., École de sciences infirmières Ingram, Université McGill, Montréal, QC

Erin Cook, inf. aut., M.Sc., Infirmière-chef, Clinique d'oncologie, CIUSSS du Centre-Ouest-de-l'île-de-Montréal, Clinique d'oncologie, Centre du cancer Segal, Montréal, QC

Lucie Tremblay, inf. aut., B.Sc.inf., Infirmière clinicienne, assistante infirmière-chef, CIUSSS du Centre-Ouest-de-l'île-de-Montréal, Clinique d'oncologie, Centre du cancer Segal, Hôpital général juif, Montréal, QC

Carmen G. Loiselle*, inf. aut., Ph.D., Professeure, Département d'oncologie, École de sciences infirmières Ingram, Université McGill, Co-directrice universitaire et chercheuse principale, Centre du cancer Segal, Hôpital général juif, 3755, Chemin de la Côte-Sainte-Catherine, Pav. E-748, Montréal, Québec H3T 1E2

514-340-8222, poste 23940, carmen.g.loiselle@mcgill.ca

*auteure-ressource

DOI: $10.5737 / 23688076283172177$
Implications pour la pratique infirmière : En plus des éléments considérés comme cruciaux pour accroître le sentiment de proximité, les prochaines études devront continuer à documenter l'influence de l'interaction entre les facteurs démographiques, physiques/psychologiques et culturels sur cette perception.

\section{CONTEXTE}

$\mathrm{E}$ n général, un diagnostic de cancer a de lourdes répercussions sur la vie des patients et de leurs familles. Pour les personnes traitées contre un cancer, l'infirmière primaire représente bien souvent la principale source d'encadrement et de soutien. Prodiguer ce type de soins exige de comprendre parfaitement les besoins du patient ainsi que ses préférences physiques et psychologiques.

\section{Soins centrés sur la personne}

On définit les soins centrés sur la personne comme des soins respectueux et adaptés aux préférences, besoins et valeurs du patient, valeurs sur lesquelles s'appuient toutes les décisions prises au sujet des soins cliniques (Balogh et al., 2011). De même, le nouveau concept de « patients partenaires » dans le domaine des soins considère le patient comme un membre de son équipe soignante (Pomney, Ghadiri, Karazivan, Fernandez et Clavel, 2015). Pour répondre aux besoins du patient, il est bien reconnu que les concepts de patients partenaires et de soins centrés sur la personne représentent des éléments fondamentaux pour offrir des soins oncologiques complets (Arora, Street, Epstein et Butow, 2009; Balogh et al., 2011; National Cancer Institute, 2007; Kvåle et Bondevik, 2008). En pratique, cela consiste être à l'écoute des questions, des besoins et des opinions du patient puis en faire part à l'équipe soignante. Le patient reste ainsi informé sur sa maladie et bénéficie d'une approche basée sur les objectifs, dans le respect de ses désirs et capacités (Flagg, 2015). Une fois mises en place, ces approches augmentent la satisfaction et la confiance du patient envers l'équipe de soins (Thórarinsdóttir et Kristjánsson, 2013).

\section{Le sentiment de proximité}

Le sentiment de proximité, tel que décrit par Thorne et collègues (2005), contribue à améliorer la qualité des soins lorsquel'on veut qu'ils soient adaptés aux caractéristiques et aux besoins uniques des patients, en privilégiant le contact humain. Ce concept, basé sur la reconnaissance, la compréhension et le respect des préférences et de l'identité du patient, gagne en importance dans le domaine des soins centrés sur la personne. (Fillion, de Serres, Cook, Goupil, Bairati et Doll, 2009; Jacobsen, Bouchard, Emed, Lepage et Cook, 2015; Thorne, Kuo, Armstrong, McPherson, Harris et Hislop, 2005). 
L'approche personnalisée adoptée par les prestataires de soins $\mathrm{du}$ cancer repose sur la représentation du patient dans son unicité. Adaptée aux objectifs, aux sentiments et aux expériences du patient, cette approche permet de tisser des liens entre le patient et l'équipe de soins (Fillion et al., 2009; Jacobsen et al., 2015). Malheureusement, les recherches montrent que les patients qui évoluent dans les milieux de soins oncologiques ont souvent l'impression qu'on ignore leurs besoins et qu'on tient peu compte de leurs préoccupations (Brataas, Thorsnes et Hargie, 2010). Pour remédier à cette situation, les cliniciens peuvent améliorer l'expérience du patient en s'intéressant à ses objectifs et à ses besoins, en respectant ses croyances et valeurs, et en étant sensibles aux autres facettes de son quotidien (Kruijver, Kerkstra, Bensing et van de Wiel, 2000; Mazor et al., 2013; Thomsen, Pedersen, Johansen, Jensen et Zachariae, 2007). En tant que membres clés de l'équipe soignante, les infirmières occupent un rôle important dans la prestation de soins personnalisés aux patients atteints de cancer. Cette étude se penche sur la façon dont les patients suivis dans un milieu de chimiothérapie ambulatoire achalandé définissent leur sentiment de proximité.

\section{MÉTHODOLOGIE}

\section{Devis de létude, échantillon et contexte}

Pour répondre aux principaux objectifs de l'étude, des descriptions exhaustives du phénomène d'« intérêt » ont été recherchées. Pour ce faire, un devis descriptif qualitatif permettant une exploration approfondie du sujet a été utilisé. Le guide d'entrevue élaboré par les auteures intégrait des questions ouvertes afin d'obtenir plus de détails sur l'expérience des patients, et s'inspirait des écrits trouvés sur le sentiment de proximité patient-infirmière ainsi que de la recherche portant sur les interactions entre ces deux parties. En voici quelques exemples: Qu'est-ce qui vous fait penser que votre infirmière vous connaît bien? Comment décrivez-vous votre expérience concernant l'approche personnalisée adoptée par votre infirmière? Sentez-vous que les soins que vous avez reçus ont été adaptés pour répondre à vos besoins?

Les participants ont été recrutés dans un centre de cancérologie ambulatoire affilié à une université de Montréal, au Québec, du 1er septembre 2016 au 31 décembre 2016. Un échantillonnage de convenance a été utilisé pour recruter dix participants qui répondaient à ces critères d'inclusion : 1) recevoir ou avoir reçu des traitements contre le cancer dans ce centre; 2) être âgé de 18 ans ou plus; et 3) être capable de converser et de lire en anglais ou en français. Les patients étaient exclus si : 1) ils étaient suivis par le personnel de l'unité de recherche clinique; ou 2) ils présentaient une limitation cognitive ou physique (douleur intense ou nausée, par exemple) susceptible de nuire à leur pleine participation à l'étude. Parmi les 20 participants recrutés au départ, 3 n'ont pu être joints (abandon après 3 tentatives), 3 ont annulé leur rendez-vous, 1 a été exclu parce qu'il ne parlait ni français ni anglais, et 3 autres n'ont pu compléter leur entrevue avant la date limite imposée par l'université. L'échantillon final $(\mathrm{N}=10)$ était composé de patients dont les traitements de chimiothérapie étaient en cours $(\mathrm{n}=9)$ ou avaient été complétés $(\mathrm{n}=1)$ dans le centre de cancérologie ambulatoire (le tableau 1 présente les données démographiques). L'échantillon était

\begin{tabular}{|c|c|}
\hline Tableau 1. Caractéristiques & \\
\hline & $n$ \\
\hline Sexe & \\
\hline Masculin & 6 \\
\hline Féminin & 4 \\
\hline Âge & \\
\hline $20-40$ ans & 1 \\
\hline $41-60$ ans & 2 \\
\hline $61-80$ ans & 6 \\
\hline $81-90$ ans & 1 \\
\hline Types de cancer (Primaire) & \\
\hline Testiculaire & 1 \\
\hline Colorectal & 4 \\
\hline Sein & 2 \\
\hline Rénal & 1 \\
\hline Hématologique & 2 \\
\hline Stades du cancer & \\
\hline Stade I & 0 \\
\hline Stade II & 2 \\
\hline Stade III & 3 \\
\hline Stade IV & 3 \\
\hline Inconnu & 2 \\
\hline Temps écoulé depuis le dią & \\
\hline$<6$ mois & 3 \\
\hline 6-12 mois & 3 \\
\hline$>12-24$ mois & 3 \\
\hline$>24$ mois & 1 \\
\hline Nombre approximatif de vi & \\
\hline$<5$ visites & 1 \\
\hline $5-10$ visites & 5 \\
\hline $11-15$ visites & 0 \\
\hline $16-20$ visites & 4 \\
\hline Traitement & \\
\hline Traitements en cours & 9 \\
\hline Traitements complétés & 1 \\
\hline
\end{tabular}


formé d'hommes $(n=6)$ et de femmes $(n=4)$ atteints de différents types de cancer : sein $(n=2)$, colorectal $(n=4)$, hématologique $(n=2)$, testiculaire $(n=1)$ et rénal $(n=1)$. Les participants étaient âgés de 20 à 90 ans, 3 d'entre eux avaient 60 ans ou moins et 7 avaient plus de 60 ans. Tous ont déclaré n'avoir jamais été traités pour un cancer auparavant. Au moment d'être recrutés, les patients avaient reçu leur diagnostic depuis moins de 6 mois $(n=3), 6$ à 12 mois $(n=3), 13$ à 24 mois $(n=3)$ et 15 ans $(n=1)$. Fait à noter, ce milieu ambulatoire privilégie une approche de soins primaires, où une même infirmière est responsable du suivi d'un patient pendant le traitement actif de son cancer. En l'absence de cette infirmière primaire, certains jours de semaine, une autre infirmière est alors affectée aux soins.

Les participants ont été recrutés par l'entremise de bénévoles de soutien communautaire pour le cancer ou de chercheurs, qui ont brièvement présenté l'étude aux patients admissibles pour qu'ils consentent à ce que l'équipe de recherche les contacte. Les chercheurs ont ensuite rencontré les patients intéressés, revu le formulaire de consentement avec eux et, après autorisation du patient, mené une entrevue exhaustive à l'endroit et à l'heure fixée d'un commun accord.

\section{Collecte de données et analyse}

Les renseignements ont été recueillis dans le cadre d'entrevues semi-structurées qui se déroulaient en personne. Après l'entrevue, les participants ont rempli un questionnaire de données sociodémographiques. Les entrevues audio-enregistrées ont été transcrites au cours de la semaine suivant l'entrevue. La vérification des participants a été effectuée par les auteures durant les entrevues afin de clarifier les données obtenues et de confirmer si l'interprétation des réponses était juste. Suite à l'entrevue, les participants ont reformuler ou préciser leurs idées concernant les réponses fournies. Ce compte rendu a permis de vérifier la compréhension de l'intervieweur quant aux problématiques rapportées, en plus d'assurer la crédibilité et la fiabilité des données récoltées.

\section{RÉSULTATS}

Une analyse thématique a permis de catégoriser les éléments clés se rapportant à la façon dont les participants décrivaient le sentiment d' « être connu ». Le regroupement des données en thèmes a facilité l'analyse et précisé la représentation des résultats (Boyatzis, 1998).

$\mathrm{Au}$ départ, les participants trouvaient difficile d'expliquer ce qui renforçait leur sentiment de proximité. Par exemple, ils demandaient souvent de répéter la question ou de préciser l'objectif des entrevues. Plusieurs participants ont partagé des situations concrètes vécues pour illustrer leurs expériences. De ces expériences, des énoncés intéressants concernant des interactions fructueuses ont émergé. Les comptes rendus textuels ont ensuite été révisés en fonction de ces exemples, puis nous avons relevé les similarités et différences. Les éléments semblables ont été regroupés, ce qui a mené à la formulation de thèmes plus larges : 1) se sentir accueilli dans le milieu de soins; 2) bénéficier de soins adaptés à la personne et à la situation; et 3) être approché comme une personne avec des champs d'intérêt professionnel et social avant d'être vu comme un « malade ».

\section{Se sentir accueilli dans le milieu de soins oncologiques}

Les patients ont indiqué que le sentiment d'accueil repose sur plusieurs facteurs, comme l'attitude générale des membres de l'équipe soignante, les interactions positives avec les infirmières et les bénévoles, et le fait de ne pas «se sentir comme un numéro ». Les patients recherchent et apprécient chez l'équipe soignante une attitude positive et amicale, empreinte de compassion et de respect; cette approche nourrit leur sentiment de proximité avec l'équipe. Les salutations et la familiarité contribuent à dégager de la sympathie. Comme le rapportait un participant : «[Les infirmières] me reconnaissent quand j'arrive, et je les reconnais moi aussi. » (P7) Dans le même ordre d'idées, un autre participant a souligné que l'on peut résumer le sentiment de proximité par une simple approche amicale : "Sourire aux gens, leur parler un peu, savoir qui ils sont, les saluer, c'est tout. » (P15)

Un autre participant a décrit comment l'attitude du personnel infirmier a contribué au sentiment de prise en charge : "C'est personnel, une sorte de chaleur humaine. Je me sens alors entre bonnes mains avec mon infirmière. " (P5) Plusieurs patients se sont sentis à l'aise dans le milieu de soins parce qu'ils pouvaient partager des histoires personnelles avec leur infirmière responsable, et qu'elle en faisait autant. Lorsqu'on lui a demandé ce qui contribuait au sentiment de proximité avec son infirmière, un participant a répondu: "Eh bien, s'intéresser à la famille, vous savez, l'écouter me parler de sa famille, de sa vie. Se sentir un peu plus proche, j'imagine. En savoir un peu plus l'un sur l'autre. » (P5) Une autre participante a partagé à quel point le sentiment de proximité ressenti était lié au fait qu'elle connaissait un peu mieux le passé et la vie personnelle de son infirmière (P2). Le partage d'expériences familiales similaires était aussi important pour certains participants, les faisant se sentir plus proche de leur infirmière.

Le participant 1 a décrit son expérience ainsi : «C'est un être humain, tout comme le patient. » Un patient a expliqué comment parler de la famille l'avait mené à tisser des liens avec son infirmière. Cela est arrivée en parlant de leurs enfants à chaque visite clinique : "[Mon infirmière] a de jeunes garçons et m'en parle, et elle sait que j'en ai un aussi. On en jase donc à chaque rencontre. » (P5). De même, un autre participant a senti une plus grande proximité avec les membres de son équipe soignante parce qu'ils parlaient sa langue. Il a expliqué cela en disant : « Je peux mieux expliquer les choses avec [mon infirmière]. Et il y en a deux autres aussi d'origine latine, à qui je parle et qui me rassurent. Elles sont toujours à mon écoute et je l'apprécie. » (P19)

Le lien entre les approches personnelles et impersonnelles des professionnels de la santé et le sentiment pour les participants d'être accueilli dans le milieu de soins oncologiques a été abordé. Afin de définir son sentiment de familiarité, un participant a raconté combien son expérience précédente dans un autre centre contrastait avec son expérience actuelle. "J'entrais et je me sentais comme du bétail, c'était si impersonnel. Très moderne et luxueux et tout ça, mais chose certaine, je me sentais comme un numéro là-bas. Ici, ce n'est pas comme ça, c'est plus personnalisé. Il ne s'agit pas de seulement vouloir passer au patient suivant... Même s'il $y$ a beaucoup de patients à voir, comme partout ailleurs, il semble 
régner ici une approche axée sur la personne. Dès qu'on entre ici, on sent que c'est différent. » (P11)

Cette perception et ses effets ont aussi été rapportés par un autre participant "Elle ne me traite pas comme un numéro, elle connaît mon histoire et ça, c'est très rassurant. » (P7)

\section{Recevoir des soins adaptés à la personne et à la situation}

Dans le cadre de cette étude, les « soins adaptés à la personne » résument la façon dont les prestataires de soins de santé s'adaptent aux besoins physiques et psychologiques changeants du patient. La majorité des participants $(n=9)$ considèrent que les soins adaptés à la personne et au contexte renforcent leur sentiment de proximité. Divers exemples sont venus illustrer comment l'équipe de soins a su personnaliser le plan de traitement en fonction de la situation du patient, rendre la communication transparente pour toutes les parties en jeu et offrir des soins complets et adéquats.

Les participants ont décrit à quel point la coordination du plan de traitement répondait à leurs besoins et exigences thérapeutiques. L'un d'entre eux a mentionné la façon dont l'infirmière a ajusté son intervention pour faciliter la gestion des symptômes et du traitement à la maison : "Elle a accroché la bouteille portative à l'intérieur pour éviter que le fil ne s'accroche sur une chaise, par exemple. » (P1)

Lorsqu'on lui a demandé de quelle façon il s'était senti important pour son infirmière, un participant a expliqué comment elle avait contrôlé sa pression sanguine pour déterminer le moment où il faudrait prendre la médication anti-hypertensive. «Je ne la prends plus [avant la chimiothérapie]... alors maintenant ma pression est un peu haute, elle s'en est inquiétée elle a appelé mon médecin, qui a dit de ne pas s'en faire, de continuer à surveiller le tout. » (P7)

Selon les participants, la cueillette d'information et les réponses aux questions en temps opportun étaient des aspects importants pour adapter les soins à la personne et au contexte. Un participant a partagé ceci : "Ils savent que l'on a peur. La première fois que je suis venu ici, j'étais pétrifié. Elle m'a permis de me détendre en me disant "relaxez" et en expliquant les étapes à venir. "Vous n'avez qu'à suivre ses consignes et vous passerez à travers”. » (P1). De la même manière, une autre participante a décrit sa première journée et ce que l'infirmière a fait pour l'informer et la guider : « [Elle] me donnait beaucoup d'informations sur les effets de la chimiothérapie et quoi faire ou ne pas faire, ce que j'allais ressentir, s'assurait que je comprenais, me renseignait à propos des médicaments et des effets indésirables, et des choses que j'avais besoin de savoir... Elle m'a aussi rassurée dès le début en m'offrant de la contacter en cas de besoin ou d'inquiétude, et m'a remis sa carte avec son numéro de téléphone. »(P17)

De plus, les participants ont relevé la rapidité avec laquelle l'infirmière répondait à leurs questions en dehors des visites à la clinique. L'un d'entre eux se souvient avoir contacté son infirmière pour l'interroger au sujet de ses symptômes. « La semaine dernière, j'ai eu un problème et je l'ai appelée, et en moins d'une demi-heure, elle m'avait rappelé. » (P1)

Les patients rencontrent différents professionnels de la santé, et plusieurs ont parlé de l'importance d'une communication transparente pour des soins adaptés à la personne et au contexte. Un patient a décrit de quelle façon ses symptômes avaient été communiqués au reste de l'équipe soignante par l'entremise de l'infirmière : «Quand j'ai rencontré mon oncologue, il m'a remis le dossier pour que je l'apporte à la pharmacie et c'est là que j'ai remarqué l'écriture de mon infirmière, qui rapportait la discussion que nous avions eue ensemble. Elle avait écrit que j'avais la diarrhée, que ça me mettait à l'envers et m'avait déprimé. Elle avait tout bien rempli pour que mon médecin soit au courant. » (P1)

Par ailleurs, une participante a elle aussi décrit combien elle avait apprécié la transmission efficace des informations entre les infirmières alors que son infirmière primaire était absente : "Même l'infirmière remplaçante semblait me connaître... Je lui ai dit "habituellement mon intraveineuse brûle", et elle m'a répondu "oui, je sais, mais celle-ci ne devrait pas brûler, car il y a deux médicaments en moins"... Elle avait donc déjà reçu cette information, elle le savait déjà. » (P17)

Un autre participant a décrit comment son infirmière primaire avait porté attention quand le fait d'être vu par différents membres de l'équipe l'avait inquiété : «Ils doivent avoir passé des heures [avec moi] afin de savoir quelle approche fonctionnerait avec moi. Ça m'a beaucoup aidé parce que j'étais toujours très nerveux lorsque [mon infirmière] s'absentait... mais s'ils sont formés, ils sont au courant. [Mon infirmière dirait] "Si je suis absente, ne vous inquiétez pas, mes collègues prendront soin de vous”. » (P19)

Les participants ont senti qu'ils recevaient des soins complets et personnalisés, ce qui ajoutait à leur sentiment de proximité. Concernant les soins adaptés, une participante a expliqué comment l'infirmière a su déceler des problématiques particulières à cause de la thérapie intraveineuse (IV) et a continué à adapter ses soins lors des visites suivantes. « Elle connaît déjà la situation, elle sait que l'IV sera plus difficile pour moi, alors elle prépare mon bras, elle fait toutes ces choses à l'avance, car elle sait que l'IV brûle, elle prévoit un deuxième cathéter, elle est prête... » (P17)

Un autre participant est allé dans le même sens en racontant que son infirmière avait pensé à lui donner un verre d'eau avec ses médicaments parce qu'il avait déjà eu des difficultés avec une bouteille. "J'avais pris mes médicaments avec une bouteille d'eau. Vingt pilules en tout, alors j'en avale 5 à la fois, mais certains étaient passés dans la bouteille. J'ai quand même réussi à tous les avaler. Mais à ma visite aujourd'hui, elle s'est souvenue de ce qui s'était passé la dernière fois. Un mois plus tard! J'ai été impressionné qu'elle s'en souvienne. » (P7)

Être reconnu en tant que personne ayant des champs d'intérêt professionnel et social qui vont au-delà du rôle de « malade »

Pour certains patients $(n=3)$, être reconnu comme une personne et non comme un «malade » a favorisé leur sentiment de proximité avec l'équipe de soins. Ce sentiment naissait lorsque le personnel soignant écoutait et se souvenait du contexte social du patient : éléments sur sa famille, étapes et expériences marquantes de sa vie, vie sociale et professionnelle, etc. Une participante a parlé de la compréhension de son système familial par son infirmière : "C'est comme se rappeler, vous savez, de qui est son conjoint, que l'on a un enfant de 
10 ans, ce genre de choses, c'est être "connu". » (P5) Un participant a souligné que son infirmière savait que sa femme avait été hospitalisée, un événement important pour la famille. "Quand ma femme est revenue, l'infirmière l'a serrée très fort contre elle et l'a embrassée. Ce n'est pas tout le monde qui ferait ça. » (P1)

Concernant l'idée de savoir qui on est à l'extérieur du milieu de soins, un participant a mentionné : «C'est très important vous savez, parce qu'on est plus que des patients qui reçoivent des traitements, on est aussi des personnes qui ont différents rôles... ils savent que je suis la mère de jumeaux, que je suis une épouse, une collègue, que j'ai tous ces chapeaux. » (P17)

Un autre participant a aussi parlé de l'importance d'être traité comme une personne normale par le personnel. « $\mathrm{Ce}$ n'est pas parce que je suis malade que je suis différent, vous comprenez? Je suis comme tout le monde. Pas différent... Bien sûr, je passe de longues journées ici, cinq jours par semaine, une semaine par cycle, mais ils ne vous traitent pas comme un malade, mais comme une personne normale. » (P15)

\section{DISCUSSION}

Les récits des participants à cette étude portant sur le sentiment de proximité ont fait ressortir l'importance du rôle de l'infirmière et de son approche compétente et adaptée, empreinte de chaleur humaine et de bienveillance pour l'être humain, au-delà de sa maladie. D’autres perceptions de proximité similaires ont été rapportées dans les écrits. Une étude menée par Jacobsen et collègues (2015) révèle que de jeunes adultes suivis dans un centre de cancérologie se sont sentis mieux traités parce que leur situation particulière de jeunes adultes combattant le cancer avait été prise en considération. Un personnel accueillant et amical est un autre facteur ayant contribué au sentiment de proximité avec l'équipe soignante. En outre, l'étude de Thorne et collègues (2005) portant sur la relation patient-soignant a permis d'en identifier ces principales caractéristiques : connecter humainement par le toucher physique, voir au-delà de la maladie du patient et reconnaître son unicité. Ces éléments ont aussi contribué à la satisfaction générale du patient. En explorant les effets se rapportant au sentiment de proximité, Flickinger, Saha, Moore et Beach (2013) ont indiqué que ces éléments étaient fortement associés à une meilleure participation et adhésion du patient à son traitement contre le VIH. Ces constats illustrent aussi comment les principales caractéristiques liées au sentiment de proximité contribuent à la perception de recevoir des soins de meilleure qualité.

Dans la présente étude, le sentiment de proximité se caractérisait surtout par l'adaptation des soins à la personne et à sa situation ainsi que l'échange d'information entre le patient et son infirmière primaire. Afin de prodiguer des soins adéquats, il est essentiel que les infirmières connaissent les caractéristiques uniques de leurs patients, ainsi que leurs besoins et préférences en termes de soins. Ce savoir doit servir à éclairer la prise de décisions et les interventions cliniques. La documentation scientifique récemment parue décrit les soins adaptés à la situation du patient comme une façon de « connaître le patient ", bien que la prémisse demeure la même (Radwin, 1995; Radwin, 1996; Whittmore, 2000). Lorsque les professionnels de la santé ont une connaissance approfondie $\mathrm{du}$ patient en tant que personne, ils peuvent mieux personnaliser leurs interventions. Coordonner les soins avec le pharmacien, administrer le traitement de façon à éviter un effet indésirable lié à la médication et fournir des renseignements pertinents sont ces interventions qui font que les infirmières de la présente étude ont prouvé à leurs patients qu'elles avaient compris ce qui était important et particulier dans les soins à leur prodiguer. En effet, ces interventions infirmières ont permis aux patients de se sentir proches d'elles et de devenir ensemble des partenaires dans leurs propres soins.

Avec des conclusions similaires, Radwin (2000) mentionne que « du point de vue du patient, des soins d'excellente qualité reposent sur les connaissances professionnelles, la continuité, l'écoute, la coordination, le partenariat, l'individualisation, la relation et l'empathie » (p. 179). Plus précisément, les soins infirmiers de qualité décrits par les patients englobent les attitudes positives, les connaissances et les compétences professionnelles de l'équipe d'infirmières (Attree, 2001; Rchaidia, de Casterlé, Deblaeser et Gastmans, 2009). Rchaidia et collègues (2009) notent aussi que les patients reçoivent des soins de qualité lorsque les infirmières sont pleinement conscientes des besoins des patients et qu'elles s'adaptent à l'évolution de ces besoins en offrant du soutien émotionnel, en démontrant une maîtrise des compétences techniques, en étant près du patient et en créant un environnement agréable et basé sur une certaine familiarité. Dans le cadre de cette étude, les participants ont décrit le sentiment de proximité comme faisant partie intégrante des soins infirmiers de qualité.

De même, les soins axés sur le patient se fondent sur la personnalisation des soins en fonction du profil et des besoins de l'individu (Arora et al., 2009; Ekman et al, 2011; National Cancer Institute, 2007). McCormack et McCance (2006) précisent que les soins centrés sur le patient nécessitent de prendre en consideration ses croyances et valeurs, l'implication du patient dans les soins, la prise de décision en commun, une présence compatissante, et la satisfaction des besoins physiques du patient. Le Health Innovation Network of South London (2016) présente les éléments clés des soins axés sur le patient qui sont les plus susceptibles d'entraîner des résultats positifs chez le patient. Reconnaître l'individualité du patient, favoriser une approche holistique pour l'évaluation et les soins, privilégier la coordination et la continuité des soins, créer un environnement favorable sur les plans physique, culturel et psychosocial et s'assurer que l'équipe soignante soit compétente et communicative en font partie. Nombre de ces éléments correspondent à ce que les patients ont identifié comme contributoire au sentiment de proximité.

Un troisième concept, être reconnu en tant que personne ayant des champs d'intérêt professionnel et social qui vont au-delà du « rôle de malade », a été rapporté par certains patients. Or, le sentiment de proximité avec l'équipe soignante est un élément qui a été plutôt négligé jusqu’à présent. Même si les études portant sur la proximité patient-soignant, sur les soins centrés sur les patients et sur l'idée de « connaître le patient » traitent de l'individualité et de l'unicité du patient, cela a été fait dans le but d'offrir des soins adaptés (Flagg, 2015; Radwin, 1995, 1996; Thorne et al., 2005). La présente étude a élargi la signification d'unicité et illustré la connexion avec la 
perception de proximité avec l'équipe de soins infirmiers que peuvent développer les patients. Cela implique également de reconnaître le patient en tant que personne, sans égard à la maladie. À cet effet, la reconnaissance des rôles social et professionnel du patient et le fait que le personnel clinique porte attention aux événements importants de sa vie peuvent renforcer son sentiment de proximité avec l'équipe soignante.

\section{LIMITES}

Cette étude comporte plusieurs limites. Léchantillon est homogène démographiquement, ne comprenant que des participants d'origine caucasienne qui avaient tous un conjoint et un revenu familial élevé (> 100000 \$). Cette homogénéité pourrait expliquer la similarité dans les expériences et besoins rapportés, qui pourraient différer chez les patients présentant une autre profil sociodémographique. D'autre part, la nature plutôt abstraite du concept de « proximité » pourrait avoir nui à la compréhension et à l'exploration des définitions associées au concept.

Cette étude présente le sentiment de proximité ressenti par les patients envers le personnel soignant comme un facteur clé améliorant l'expérience de traitement. On y traite aussi du

\section{RÉFÉRENCES}

Arora, N.K., Street, R.L., Jr., Epstein, R.M., \& Butow, P.N. (2009). Facilitating patient-centered cancer communication: A road map. Patient Education a Counseling, 77(3), 319-321 313p. doi:10.1016/j. pec.2009.11.003

Attree, M. (2001). Patients' and relatives' experiences and perspectives of 'good'and 'not so good' quality care. Journal of Advanced Nursing, 33(4), 456-466.

Balogh, E.P., Ganz, P.A., Murphy, S.B., Nass, S.J., Ferrell, B.R., \& Stovall, E. (2011). Patient-centered cancer treatment planning: Improving the quality of oncology care. Summary of an Institute of Medicine workshop. The Oncologist, 16(12), 1800-1805.

Boyatzis, R.E. (1998). Transforming qualitative information: Thematic analysis and code development. Sage.

Brataas, H.V., Thorsnes, S.L., \& Hargie, O. (2010). Themes and goals in cancer outpatient-cancer nurse consultations. European Journal of Cancer Care, 19(2), 184-191.

Ekman, I., Swedberg, K., Taft, C., Lindseth, A., Norberg, A., Brink, E., ... Lidén, E. (2011). Person-centered care-Ready for prime time. European Journal of Cardiovascular Nursing, 10(4), 248-251.

Fillion, L., de Serres, M., Cook, S., Goupil, R.L., Bairati, I., \& Doll, R. (2009). Professional patient navigation in head and neck cancer. Paper presented at the Seminars in Oncology Nursing.

Flagg, A.J. (2015). The role of patient-centered care in nursing. Nursing Clinics of North America, 50(1), 75-86 12p. doi:10.1016/j. cnur.2014.10.006

Flickinger, T.E., Saha, S., Moore, R.D., \& Beach, M.C. (2013). Higher quality communication and relationships are associated with improved patient engagement in HIV care. Journal of Acquired Immune Deficiency Syndromes, 63(3), 362.

Health Inovation Network. (2016). What is person-centred care and why is it important? (pp. 1-5, Publication). South London, UK. Retrieved from http://www.hin-southlondon.org/resources/ what-is-person-centred-care

Jacobsen, S., Bouchard, G., Emed, J., Lepage, K., \& Cook, E. (2015). Experiences of "being known" by the healthcare team of young adult patients with cancer. Oncology Nursing Forum, 42(3), 250-256.

Kruijver, I.P., Kerkstra, A., Bensing, J.M., \& van de Wiel, H.B. (2000). Nurse-patient communication in cancer care. A review of the literature. Cancer Nursing, 23(1), 20-31. rôle primordial joué par les infirmières dans la prestation de soins centrés sur le patient. À la lumière de ces observations, la direction des soins infirmiers au site de l'étude a instauré des réunions d'étude de cas afin de répondre à ces besoins. Les futures études devraient se pencher sur les facteurs sociodémographiques pouvant affecter le sentiment de proximité entre le patient et son équipe soignante. D’autres études pourraient aussi être menées pour élucider la façon dont les interventions infirmières particulières telles que les réunions d'étude de cas ou le recours à un plan de soins thérapeutiques infirmiers peuvent contribuer au sentiment de familiarité.

\section{REMERCIEMENTS}

Nous souhaitons remercier les bénévoles de L'espoir c'est la vie pour avoir généreusement offert de leur temps pour le recrutement des patients, ainsi que le centre de traitement du cancer de l'HGJ pour leur aide dans l'organisation des entrevues avec les participants. Les $D^{\text {res }}$ Ariella Lang et Margaret Purden ainsi que $M^{\text {mes }}$ Saima Ahmed et Jacqueline Vachon ont fourni des rétroactions fort appréciées sur les versions précédentes de cet article.

Kvåle, K., \& Bondevik, M. (2008). What is important for patient centred care? A qualitative study about the perceptions of patients with cancer. Scandinavian Journal of Caring Sciences, 22(4), 582-589.

Mazor, K.M., Beard, R.L., Alexander, G.L., Arora, N.K., Firneno, C., Gaglio, B., ... Gallagher, T.H. (2013). Patients' and family members' views on patient-centered communication during cancer care. Psycho-Oncology, 22(11), 2487-2495 2489p. doi:10.1002/pon.3317

McCormack, B., \& McCance, T.V. (2006). Development of a framework for person-centred nursing. Journal of Advanced Nursing, 56(5), 472-479.

National Cancer Institute (2007). Patient-centered communication in cancer care: Promoting healing and reducing suffering (07-6225). Bethesda, MD.

Pomney, M-P., Ghadiri, D.P., Karazivan, P., Fernandez, N., \& Clavel, N. (2015). Patients as partners: A qualitative study of patients' engagement in their health care. PLoS ONE, 10(4), e0122499.

Radwin, L.E. (1995). Knowing the patient: a process model for individualized interventions. Nursing Research, 44(6), 364-370.

Radwin, L.E. (1996). 'Knowing the patient': A review of research on an emerging concept. Journal of Advanced Nursing, 23(6), 1142-1146.

Radwin, L.E. (2000). Oncology patients' perceptions of quality nursing care. Res Nurs Health, 23(3), 179-90.

Rchaidia, L., Dierckx de Casterlé, B., De Blaeser, L., \& Gastmans, C. (2009). Cancer patients' perceptions of the good nurse: A literature review. Nursing Ethics, 16(5), 528-542.

Thomsen, D.K., Pedersen, A.F., Johansen, M.B., Jensen, A.B., \& Zachariae, R. (2007). Breast cancer patients' narratives about positive and negative communication experiences. Acta Oncologica, 46(7), 900-908 909p.

Thórarinsdóttir, K., \& Kristjánsson, K. (2013). Patients' perspectives on person-centred participation in health care: A framework analysis. Nursing Ethics, 0969733013490593.

Thorne, S.E., Kuo, M., Armstrong, E.-A., McPherson, G., Harris, S.R., \& Hislop, T.G. (2005). Being known: patients' perspectives of the dynamics of human connection in cancer care. PON PsychoOncology, 14(10), 887-898.

Whittemore, R. (2000, Jan. 1). Consequences of not "knowing the patient". Clinical Nurse Specialist, 14(2), 75-81. 


\title{
Potential mediators of improvement in painful chemotherapy-induced peripheral neuropathy via a web-based cognitive behavioural intervention
}

\author{
by Robert Knoerl, Debra L. Barton, Janean E. Holden, John C. Krauss, Beth LaVasseur, and Ellen M.L. Smith
}

\begin{abstract}
Purpose: Preliminary evidence suggests that a self-guided cognitive and behaviourally-based pain management intervention (PROSPECT) is effective for chronic painful chemotherapy-induced peripheral neuropathy (CIPN), but its mechanism of action is unknown. The purpose of this secondary analysis was to explore if changes in anxiety, depression, sleep-related impairment, or fatigue mediated improvements in worst pain following PROSPECT in individuals with chronic painful CIPN.
\end{abstract}

Methods: Sixty participants were randomized to receive self-guided cognitive behavioural pain management (access for eight weeks) or treatment as usual. A seven-day worst CIPN pain diary and the PROMIS measures of anxiety, depression, fatigue, and sleep-related impairment were administered pre/posttest (eight-weeks). Causal mediation analysis was used to quantify mediators of worst pain improvement.

Results: None of the hypothesized mediators had a statistically significant effect on worst pain ( $n=38)$.

\section{ABOUT THE AUTHORS \\ Corresponding Author: \\ Robert Knoerl, PhD, RN Post-Doctoral Research Fellow, Phyllis F. Cantor \\ Center for Research in Nursing and Patient Care Services, Dana-Farber Cancer Institute, 450 Brookline Avenue, LW 517, Boston, MA 02215 \\ Email: Robert_knoerl@DFCI.Harvard.edu \\ Phone: 617-632-6386 \\ Debra L. Barton, RN, PhD, AOCN, FAAN, Mary Lou Willard French Professor of Nursing, University of Michigan School of Nursing, Ann Arbor, MI Email: debbartn@umich.edu \\ Janean E. Holden, PhD, RN, FAAN, Barbara A. Therrien Collegiate Professor of Nursing, University of Michigan School of Nursing, Ann Arbor, MI Email: holdenje@umich.edu}

John C. Krauss, MD, Assistant Professor, University of Michigan Comprehensive Cancer Center, Ann Arbor, MI

Email:jkrauss@med.umich.edu

Beth LaVasseur RN, MS, Director of Oncology Research and Operation, St. Joseph Mercy Hospital, Ann Arbor, MI

Email: beth.lavasseur@st.joeshealth.org

Ellen M.L. Smith, PhD, APRN, AOCN®, FAAN, Associate Professor, University of Michigan School of Nursing, Ann Arbor, MI

Email: ellenls@umich.edu

DOI:10.5737/23688076283178183
Implications: Further research is needed to identify potential mediators of pain intensity that can be targeted by specific cognitive behavioural strategies to improve painful CIPN severity.

Key words: chronic pain, chemotherapy-induced peripheral neuropathy, cognitive behaviour therapy, peripheral nervous system disease/chemically induced

\section{INTRODUCTION}

Chronic painful chemotherapy-induced peripheral neuropathy (CIPN) occurs in $40 \%$ of individuals receiving neurotoxic chemotherapy agents such as platinums or taxanes (Kautio, Haanpää, Kautiainen, Kalso, \& Saarto, 2011; Smith, Cohen, Pett, \& Beck, 2011). Chronic painful CIPN is characterized by burning/shooting pain, numbness, and tingling in the hands and feet that can persist months to years following the completion of neurotoxic chemotherapy and may negatively affect quality of life and/or physical function (Beijers, Mols, Dercksen, Driessen, \& Vreugdenhil, 2014; Smith et al., 2014).

Currently, there is only one pharmacological (duloxetine $60 \mathrm{mg} /$ day) and no non-pharmacological treatment recommended for the management of chronic painful CIPN (Hershman et al., 2014; Smith et al., 2013). A recently conducted randomized, waitlist-controlled trial (Knoerl, Smith, et al., 2017) examined the efficacy of a self-guided online cognitive and behaviourally-based pain management intervention called Proactive Self-Management Program for Effects of Cancer Treatment (PROSPECT) on worst CIPN pain severity. Results demonstrated that PROSPECT usage significantly reduced worst pain intensity in comparison to individuals receiving usual care $(\mathrm{p}=0.046)$. Despite the statistically significant findings, only $47 \%$ of individuals receiving PROSPECT experienced at least a $10 \%$ reduction in pain. PROSPECT did not work for all participants and little is known about how cognitive behavioural pain management works to improve painful CIPN. Thus, further research is needed to identify mediators of pain intensity that can be targeted by PROSPECT to improve the intervention's effect on painful CIPN severity.

Numerous studies provide evidence supporting the relationships between several mediating variables and chronic painful CIPN intensity (i.e., anxiety, depression, fatigue, and sleep-related impairment (Hershman et al., 2016; Smith et al., 2015). Further, these relationships are supported by shared underlying pathophysiological mechanisms: 1) neurobiological changes (Boakye et al., 2016; Nudelman et al., 2016; Zhuo, 2016), 2) pronounced stress response (e.g., disruption of the hypothalamic-pituitary-adrenal axis), 3) cytokine dysregulation, and 4) 5-HT and norepinephrine dysregulation 
(Barsevick, Frost, Zwinderman, Hall, \& Halyard, 2010; Boakye et al., 2016). Cognitive behavioural pain management may work to decrease pain intensity by inducing structural changes in the brain, which subsequently may influence descending inhibitory nociceptive pathways through the release of norepinephrine and serotonin (Jensen et al., 2012; Seminowicz et al., 2013). Thus, because chronic painful CIPN co-occurs with symptoms that share similar pathophysiological mechanisms and cognitive behavioural pain management targets mechanisms that are common to all symptoms, it's possible that improvements in anxiety, depression, fatigue, or sleep-related impairment may also improve pain. However, we are unaware of published studies that have examined mediators of chronic painful CIPN pain intensity improvement following selfguided cognitive behavioural pain management. The purpose of this secondary analysis was to explore the mediating effect of mean changes in sleep-related impairment, anxiety, depression, or fatigue on worst pain intensity following PROSPECT in individuals with chronic painful CIPN.

\section{MATERIALS AND METHODS}

\section{Design, Setting, and Sample}

This current study was a secondary analysis of a pilot, randomized, wait-list controlled trial testing the effect of PROSPECT on worst CIPN pain intensity in comparison to individuals receiving treatment as usual (Knoerl et al., 2017). Briefly, 60 patients with chronic painful CIPN (e.g., $\geq 4 / 10$ worst CIPN pain for $\geq 3$ months since completion of neurotoxic chemotherapy) were recruited from five academic and/ or community outpatient cancer centres over six months. Patients were excluded if they had neuropathy due to other causes or planned to receive neurotoxic chemotherapy at any point during the study. The study protocol was approved by the study sites' Institutional Review Board and enrolled participants provided written informed consent.

\section{Measures}

An 11-point numerical rating scale ("10" represents worst pain imaginable) was used to measure worst CIPN pain severity (Cleeland \& Ryan, 1994) and was administered via a seven-day diary at the baseline and eight-week time points. Participants' responses from the seven-day diary were averaged at the respective time points. In addition, various Patient Reported Outcome Measurement Information System (PROMIS) measures were used to quantify anxiety, depression, fatigue, and sleep-related impairment in the recruited sample (Cella et al., 2007). The PROMIS Anxiety 4a (four items; $1=$ never; $5=$ always; transformed total score range 40.3-81.6) measures self-reported fearfulness, worry, and uneasiness over the past seven days (Kroenke, Yu, Wu, Kean, \& Monahan, 2014). The PROMIS Emotional Distress-Depression 4a (four items; 1 = never; 5 = always; transformed total score range 41.0-79.4) examines patient reported sadness, self-perception, loneliness, and self-purpose over the past seven days (Bartlett et al., 2015; Kroenke et al., 2014). The PROMIS Fatigue 4a (4 items; 1 = not at all; 5 = very much; transformed total score range 33.7-75.8) measures self-reported feelings of tiredness and exhaustion that likely decrease one's ability to perform daily activities and function normally in family/personal roles (Bartlett et al., 2015). The sleep-related Impairment 8a (four items; $1=$ not at all; 5 = very much; transformed total score range 30.0-80.1) measures self-reported perceptions of alertness, sleepiness, tiredness during the day, and functional impairment associated with poor sleep over the past seven days (Yu et al., 2012).

\section{Procedures}

At baseline, prior to randomization, participants completed the first day of the seven-day worst CIPN pain diary and the PROMIS subscales via computer tablet. The principal investigator then randomized participants to a study group (eight weeks of PROSPECT or treatment as usual) in a 1:1 ratio using a computer generated random numbers table. Participants then received a paper copy of the seven-day worst CIPN pain diary (to complete the remaining six days of the diary) and submitted their scores via an emailed survey link. Following completion of the pain diary, participants were emailed the link to the PROSPECT website or information about the treatment as usual control group. The PROSPECT website contains cognitive behavioural strategies (e.g., activity pacing for fatigue, progressive muscle relaxation/deep-breathing for anxiety/depression, sleep hygiene strategies for sleep-impairment) and self-management information (e.g., patient-provider communication about symptoms and goal setting) designed to help individuals manage cancer treatment-related symptoms. The content is delivered via written information and videos. The PROSPECT intervention also recommends different modules and strategies based on the participants self-reported symptoms. Participants were trained by the principal investigator at baseline about how to access/use the PROSPECT website and were encouraged to use the PROSPECT website at their discretion. Eight weeks following randomization, participants were emailed electronic versions of the seven-day worst CIPN pain intensity diary and PROMIS measures.

\section{Statistical Analyses}

$\mathrm{R}$ version 3.4.0 was utilized to analyze all data ( $R$ Development Core Team, 2017). The sample analyzed was based on individuals who completed all pre/posttest survey data in the primary study. There were 38 individuals who provided complete baseline and week eight worst CIPN pain intensity in the primary study (Knoerl et al., 2017), but only 37 of those individuals provided complete data for the secondary outcomes explored in this study $1<5 \%$ missing data for this analysis). To be consistent with the sample size of the primary study, we used mean imputation to handle missing data for one participant (Little, Jorgensen, Lang, \& Moore, 2014). We did not conduct an a priori power analysis due to the exploratory nature of this analysis.

Causal mediation (Imai, Keele, \& Tingley, 2010) was used to explore if changes in anxiety, depression, fatigue, or sleep-related impairment mediated the effects of PROSPECT on worst pain intensity improvement over the eight-week treatment period (Figure 1). The causal mediation effect is defined as the indirect effect of the treatment on the dependent variable through the mediators (Paths "A" and "B" in Figure 1) (Imai et al., 2010). The indirect effect can be further defined as the 
Hypothesized Mediators

\begin{tabular}{|c|c|}
\hline Anxiety & $\begin{array}{c}\text { Sleep-related } \\
\text { Impairment }\end{array}$ \\
\hline Depression & Fatigue \\
\hline
\end{tabular}

A

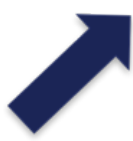

Independent Variable

$$
\text { PROSPECT }
$$

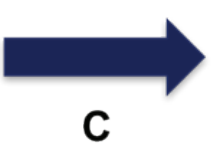

B

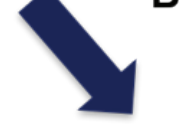

Dependent Variable

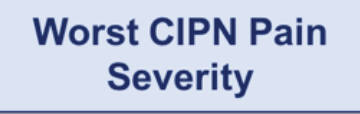

Figure 1. Hypothesized Mediation Model of PROSPECT on Worst CIPN Pain Intensity This figure describes the hypothesized mediators of the effect of PROSPECT on worst CIPN pain intensity. Paths " $\mathrm{A}$ " and "B" represent the indirect effect of PROSPECT on worst CIPN pain intensity as explained through the mediators. Path " $\mathrm{C}$ " represents the direct effect of PROSPECT on worst CIPN pain intensity.

change in the outcome (i.e., worst pain) when the value of the mediator (e.g., anxiety) is changed from the value reported by the control to the value reported by the treatment.

Descriptive statistics (mean, SD) were calculated for all continuous data at the baseline and week eight time points (i.e., worst CIPN pain, fatigue, anxiety, depression, and sleep-related impairment). Next, to assess the effect of PROSPECT on the hypothesized mediators, week eight mean scores in anxiety, depression, sleep-related impairment, and fatigue were compared between groups using ANCOVA adjusting for baseline scores. If results showed that PROSPECT had no effect on a hypothesized mediator, then mediation analyses could not be conducted because a variable can only be a mediator of an outcome when it is significantly affected by the treatment (Imai et al., 2010). Subsequently, for variables that demonstrated a statistically significant improvement following PROSPECT use, we modelled: 1) the mediators (anxiety, depression, fatigue, and/or sleep-related impairment week eight scores, respectively) given the treatment and baseline covariates (i.e., baseline worst pain scores), and 2) the outcome (worst pain intensity week eight score) given the treatment, mediator, and baseline covariates (Imai et al., 2010; Imai, Keele, Tingley, \& Yamamoto, 2015). These two models were then combined into the mediate function from the Mediation (Tingley et al., 2014) package to estimate the causal mediation effect and 95\% CI of the causal mediation effect for each mediation model.

\section{RESULTS}

\section{Sample Characteristics}

The demographic and cancer treatment-related characteristics of the recruited sample have been previously described (Knoerl, Smith, et al., 2017). The mean age of the study participants was $61.15(\mathrm{SD}=9.06$, Range $=40-78)$ years old. The sample was mainly female (75\%), Caucasian (91.7\%), college educated (82.1\%), retired (43.3\%), married $(70 \%)$, and regularly used a computer (85\%). Additionally, most participants had breast (38.3\%) or gastrointestinal (43.3\%) cancers and had varying cancer severity. Individuals receiving PROSPECT had more severe fatigue and sleep-related impairment at baseline in comparison to individuals receiving treatment as usual. Also, individuals who did not complete the study had more advanced cancer severity ( $46 \%$ had Stage IV cancer in comparison to $19 \%$ for completers), but, there were no considerable differences in baseline pain or co-occurring symptom severity between completers and non-completers.

\section{Mediation Analysis}

Table 1 describes mean scores for the variables of anxiety, depression, fatigue, and sleep - related impairment at the baseline and week eight time points. There were no significant differences in anxiety, fatigue, sleep-related impairment, or depression severity between groups at the week eight time point $(\mathrm{p}>0.05, \mathrm{n}=38$ ). Trends in anxiety, depression, and fatigue across time also indicated that PROSPECT provided no clear benefit over usual care. Trends in sleep-related impairment severity suggested that individuals receiving PROSPECT were experiencing consistent improvements as the study progressed. Nevertheless, because PROSPECT did not have a statistically significant effect on the hypothesized mediators in comparison to the treatment as usual control group, mediation analyses could not be conducted.

\section{DISCUSSION}

The results of the mediation analyses revealed that none of the hypothesized influencing factors of chronic painful CIPN significantly mediated worst CIPN pain intensity improvement following PROSPECT. Published studies have demonstrated that emotional factors mediate pain intensity improvement following in-person (i.e., anxiety) and online (i.e., stress and depression) 


\begin{tabular}{|c|c|c|c|}
\hline Outcomes $(n=38)^{a}$ & Intervention Mean (SD) & Wait-List Control Mean (SD) & Contrast Between Groups ${ }^{a}$ \\
\hline \multicolumn{4}{|c|}{ Sleep-related Impairment } \\
\hline Baseline & $58.74(6.65)$ & $55.84(5.38)$ & \multirow{2}{*}{$\begin{array}{l}\mathrm{B}=0.22 ; p=0.87 \\
\mathrm{Cl}=-2.39,2.82\end{array}$} \\
\hline Week Eight & $56.71(5.90)$ & $54.34(5.86)$ & \\
\hline \multicolumn{4}{|l|}{ Fatigue } \\
\hline Baseline & $59.59(6.55)$ & $53.31(7.84)$ & \multirow{2}{*}{$\begin{array}{l}B=0.22 ; p=0.92 \\
C l=-3.90,4.33\end{array}$} \\
\hline Week Eight & $56.83(8.41)$ & $51.47(7.92)$ & \\
\hline \multicolumn{4}{|l|}{ Depression ${ }^{b}$} \\
\hline Baseline & $52.79(7.03)$ & $47.89(7.12)$ & \multirow{2}{*}{$\begin{array}{l}B=2.54 ; p=0.16 \\
C l=-1.03,6.11\end{array}$} \\
\hline Week Eight & $52.14(6.89)$ & $46.73(6.13)$ & \\
\hline \multicolumn{4}{|l|}{ Anxiety } \\
\hline Baseline & $54.45(7.27)$ & $50.09(7.82)$ & \multirow{2}{*}{$\begin{array}{l}B=1.38 ; p=0.55 \\
C l=-3.23,5.99\end{array}$} \\
\hline Week Eight & $52.62(8.59)$ & $48.63(7.41)$ & \\
\hline
\end{tabular}

cognitive behavioural pain management (DasMahapatra, Chiauzzi, Pujol, Los, \& Trudeau, 2015; McCracken, Gross, \& Eccleston, 2002). One reason as to why anxiety and depression were not observed as mediators in this current study is that the PROSPECT intervention did not contain enough strategies to adequately address these symptoms. For example, cognitive restructuring, a key strategy of cognitive behavioral therapy for anxiety and depression, was not included in PROSPECT (Beck, 2010). Cognitive restructuring has been demonstrated to be a key component of previous self-guided cognitive behavioural pain management interventions. Of the seven-self-guided cognitive behavioural pain management trials reviewed by Knoerl, Lavoie Smith, \& Weisberg (2015), four had positive effects on anxiety/ depression. These four trials placed a specific emphasis on cognitive restructuring by providing participants with access to modules containing this strategy for multiple weeks. Conversely, programs that focused more on self-management (e.g., communication with provider, medication management, goal setting) alone were less effective for anxiety/depression. Future prototypes of PROSPECT should include and emphasize modules specific to cognitive restructuring strategies to target symptoms such as anxiety and depression.

Less is known regarding the efficacy of cognitive behavioral pain management for pain-related fatigue and sleep-related impairment in individuals with chronic pain (Knoerl, Lavoie Smith, \& Weisberg, 2015). However, there is considerable evidence supporting the use of cognitive behavioral therapy for insomnia and fatigue (Price, Mitchell, Tidy, \& Hunot, 2008; Zachariae, Lyby, Ritterband, \& O’Toole, 2016). For instance, a recent randomized controlled trial by Ritterband et al. (2017) tested a self-guided cognitive behavioural intervention for sleep that incorporated sleep hygiene, sleep restriction, stimulus control, relapse prevention, and cognitive restructuring strategies. Results suggested that individuals receiving the intervention had significantly improved insomnia severity ( $\mathrm{p}<0.001$ ) in comparison to individuals receiving insomnia education (Ritterband et al., 2017). Moreover, strategies aimed at managing and increasing physical activity have been shown to be effective for fatigue (Larun, Brurberg, OdgaardJensen, \& Price, 2016). Thus, future prototypes of PROSPECT may explore adding strategies related to sleep restriction (e.g., sleeping/waking at certain times to relearn proper sleep dynamics), cognitive restructuring strategies in the context of sleep-related impairment, and additional ways to manage and increase physical activity to target fatigue and sleep-related impairment in individuals with chronic painful CIPN.

While we assessed the mediating effect of anxiety, depression, fatigue, and sleep-related impairment on worst CIPN pain intensity, there are other influencing factors that may mediate worst CIPN pain intensity improvement that we did not measure. Specifically, cognitive variables (e.g., perceived control over pain, pain catastrophizing, and self-efficacy to manage pain) have been shown to mediate chronic pain improvement in prior research (Seminowicz et al., 2013; Turner, Holtzman, \& Mancl, 2007). PROSPECT may be modified to incorporate strategies such as cognitive restructuring (e.g., identifying and reframing automatic negative thoughts about symptoms such as pain, anxiety, depression) (Beck, 2010) to target cognitive variables such as catastrophizing in subsequent studies. 


\section{IMPLICATIONS}

The nature of this research has several implications for clinical nurses. Despite the negative results of this study, nurses should continue to encourage self-management strategy use in individuals with cancer treatment-related symptoms. Specifically, an integrative review by Hammer et al. (2015) reviewed 46 articles testing self-management interventions for individuals with cancer (all studies led by nurse-scientists) and reported that several of the reviewed self-management interventions had positive effects on cancer treatment-related symptoms (e.g., pain, fatigue, depression, anxiety) (Hammer et al., 2015). Further, self-management strategies are associated with no notable side effects and can be administered concurrently with standard treatments. However, before delivering self-management strategies, nurses must assess their patients' willingness and ability to self-manage symptoms. Subsequently, nurses must select self-management strategies that coincide with their patients' preferences/abilities and schedule (e.g., appointments, treatments, personal life) (McCorkle et al., 2011).

\section{LIMITATIONS}

There are several limitations to this study. The study was underpowered, which may increase the probability of finding a false negative result (Type II error). Participants only interacted with the PROSPECT website for eight weeks. Therefore, participants may not have had enough time to learn and incorporate the strategies from the website into their day-to-day life to influence behaviour change related to pain management. Similarly, due to the self-guided nature of the intervention, perhaps participants did not receive the optimal dose of the intervention to decrease pain-related symptoms. Lastly, while we collected data related to the amount of time participants said they spent using PROSPECT each week (Knoerl et

\section{REFERENCES}

Barsevick, A., Frost, M., Zwinderman, A., Hall, P., \& Halyard, M. (2010). I'm so tired: Biological and genetic mechanisms of cancerrelated fatigue. Quality of Life Research, 19(10), 1419-1427. http://doi. org/10.1007/s11136-010-9757-7

Bartlett, S.J., Orbai, A.-M., Duncan, T., DeLeon, E., Ruffing, V., CleggSmith, K., \& Bingham, C.O. (2015). Reliability and validity of selected PROMIS measures in people with rheumatoid arthritis. PLOS ONE, 10(9), e0138543. http://doi.org/10.1371/journal.pone.0138543

Beck, J.S. (2010). Cognitive therapy. In the Corsini Encyclopedia of Psychology. Hoboken, NJ, USA: John Wiley \& Sons, Inc. http:// doi.org/10.1002/9780470479216.corpsy0198

Beijers, A., Mols, F., Dercksen, W., Driessen, C., \& Vreugdenhil, G. (2014). Chemotherapy-induced peripheral neuropathy and impact on quality of life 6 months after treatment with chemotherapy. The Journal of Community and Supportive Oncology, 12(11), 401-6. http:// doi.org/10.12788/jcso.0086

Boakye, P.A., Olechowski, C., Rashiq, S., Verrier, M.J., Kerr, B., Witmans, M., ... Dick, B.D. (2016). A critical review of neurobiological factors involved in the interactions between chronic pain, depression, and sleep disruption. The Clinical Journal of Pain, 32(4), 327-36. http://doi.org/10.1097/ AJP.0000000000000260 al., 2017), we did not collect data about how often participants spent using specific modules within PROSPECT. Thus, it is possible that participants did not routinely use the modules containing strategies related to anxiety, depression, fatigue, and/or sleep-related impairment. Despite these limitations, the results of this study contribute to the growing body of literature surrounding the identification of mediators of pain intensity improvement following cognitive behavioral pain management to gain a greater understanding of how this treatment may work to improve pain.

\section{CONCLUSION}

In conclusion, none of the hypothesized mediators of chronic painful CIPN were significant. Due to the small sample size, the mediating effect of these co-occurring symptoms of CIPN should be reevaluated in a larger study. Further, perhaps the PROSPECT intervention should be modified in the future to amplify its effect on potential mediation targets. The identification of mediators of pain intensity in individuals with painful CIPN will allow for the targeting of behavioral strategies to factors known to improve pain intensity.

\section{DISCLOSURES}

The authors declare no conflicts of interest. This work was supported by the University of Michigan School of Nursing New Investigator Award and Rackham Graduate Student Research Grant. The funding source had no role in study design, data collection/analysis, or manuscript preparation. Creation of the PROSPECT website was supported by the Damon Runyon Cancer Research Foundation (\#CI-53-10) awarded to Norah Lynn Henry. PROSPECT was adapted from the website, Fibroguide.com, which was developed with support from Grant numbers R01-AR050044 (NIAMS/NIH), and DAMD 17-00-2-0018 (Department of Defense).

Cella, D., Yount, S., Rothrock, N., Gershon, R., Cook, K., Reeve, B., ... PROMIS Cooperative Group. (2007). The Patient-Reported Outcomes Measurement Information System (PROMIS): Progress of an NIH roadmap cooperative group during its first two years. Medical Care, 45(5 Suppl. 1), S3-S11. http://doi.org/10.1097/01. mlr.0000258615.42478.55

Cleeland, C.S., \& Ryan, K.M. (1994). Pain assessment: global use of the Brief Pain Inventory. Annals of the Academy of Medicine, Singapore, 23(2), 129-38. Retrieved from http://www.ncbi.nlm.nih. gov/pubmed/8080219

DasMahapatra, P., Chiauzzi, E., Pujol, L.M., Los, C., \& Trudeau, K.J. (2015). Mediators and moderators of chronic pain outcomes in an online self-management program. The Clinical Journal of Pain, 31(5), 404-13. http://doi.org/10.1097/AJP.0000000000000125

Hammer, M.J., Ercolano, E.A., Wright, F., Dickson, V.V., Chyun, D., \& Melkus, G.D. (2015). Self-management for adult patients with cancer. Cancer Nursing, 38(2), E10-E26. http://doi.org/10.1097/ NCC. 0000000000000122

Hershman, D.L., Lacchetti, C., Dworkin, R.H., Lavoie Smith, E.M., Bleeker, J., Cavaletti, G., ... Oncology, A.S. of C. (2014). Prevention and management of chemotherapy-induced peripheral neuropathy in survivors of adult cancers: American Society of Clinical 
Oncology clinical practice guideline. Journal of Clinical Oncology: Official Journal of the American Society of Clinical Oncology, 32(18), 1941-1967. http://doi.org/10.1200/JCO.2013.54.0914

Hershman, D.L., Till, C., Wright, J.D., Awad, D., Ramsey, S.D., Barlow, W.E., ... Unger, J. (2016). Comorbidities and risk of chemotherapyinduced peripheral neuropathy smong participants 65 years or older in Southwest Oncology Group Clinical Trials. Journal of Clinical Oncology, 34(25), 3014-3022. http://doi.org/10.1200/ JCO.2015.66.2346

Imai, K., Keele, L., \& Tingley, D. (2010). A general approach to causal mediation analysis. Psychological Methods, 15(4), 309-334. http:// doi.org/10.1037/a0020761

Imai, K., Keele, L., Tingley, D., \& Yamamoto, T. (2015). Causal mediation analysis using $R *$. Retrieved from http://imai.princeton. edu

Jensen, K.B., Kosek, E., Wicksell, R., Kemani, M., Olsson, G., Merle, J.V, ... Ingvar, M. (2012). Cognitive Behavioral Therapy increases pain-evoked activation of the prefrontal cortex in patients with fibromyalgia. Pain, 153(7), 1495-503. http://doi.org/10.1016/j. pain.2012.04.010

Kautio, A.-L., Haanpää, M., Kautiainen, H., Kalso, E., \& Saarto, T. (2011). Burden of chemotherapy-induced neuropathy-A crosssectional study. Supportive Care in Cancer, 19(12), 1991-1996. http:// doi.org/10.1007/s00520-010-1043-2

Knoerl, R., Lavoie Smith, E.M., \& Weisberg, J. (2015). Chronic pain and cognitive behavioral therapy: An integrative review. Western Journal of Nursing Research (journal article). http://doi. org/0193945915615869 [pii]

Knoerl, R., Smith, E.M.L., Barton, D.L., Williams, D.A., Holden, J.E., Krauss, J.C., \& LaVasseur, B. (2017). Self-guided online cognitive behavioral strategies for chemotherapy-induced peripheral neuropathy: A multicenter, pilot, randomized, wait-list controlled trial. The Journal of Pain. http://doi.org/10.1016/j.jpain.2017.11.009

Kroenke, K., Yu, Z., Wu, J., Kean, J., \& Monahan, P.O. (2014). Operating characteristics of PROMIS four-item depression and anxiety scales in primary care patients with chronic pain. Pain Medicine, 15(11), 1892-901. http://doi.org/10.1111/pme.12537

Larun, L., Brurberg, K.G., Odgaard-Jensen, J., \& Price, J.R. (2016). Exercise therapy for chronic fatigue syndrome. In L. Larun (Ed.), Cochrane Database of Systematic Reviews. Chichester, UK: John Wiley \& Sons, Ltd. http://doi.org/10.1002/14651858.CD003200. pub6

Little, T.D., Jorgensen, T.D., Lang, K.M., \& Moore, E.W.G. (2014). On the joys of missing data. Journal of Pediatric Psychology, 39(2), 151-162. http://doi.org/10.1093/jpepsy/jst048

McCorkle, R., Ercolano, E., Lazenby, M., Schulman-Green, D., Schilling, L.S., Lorig, K., \& Wagner, E.H. (2011). Self-management: Enabling and empowering patients living with cancer as a chronic illness. CA: A Cancer Journal for Clinicians, 61(1), 50-62. http://doi. org/10.3322/caac.20093

McCracken, L.M., Gross, R.T., \& Eccleston, C. (2002). Multimethod assessment of treatment process in chronic low back pain: Comparison of reported pain-related anxiety with directly measured physical capacity. Behaviour Research and Therapy, 40(5), 585-94. Retrieved from http://www.ncbi.nlm.nih.gov/ pubmed/12038650

Nudelman, K.N.H., McDonald, B.C., Wang, Y., Smith, D.J., West, J.D., O’Neill, D.P., ... Saykin, A.J. (2016). Cerebral perfusion and Gray Matter Changes associated with chemotherapy-induced peripheral neuropathy. Journal of Clinical Oncology: Official Journal of the American Society of Clinical Oncology, 34(7), 677-83. http://doi. org/10.1200/JCO.2015.62.1276
Price, J.R., Mitchell, E., Tidy, E., \& Hunot, V. (2008). Cognitive behaviour therapy for chronic fatigue syndrome in adults. In J.R. Price (Ed.), Cochrane Database of Systematic Reviews (p. CD001027). Chichester, UK: John Wiley \& Sons, Ltd. http://doi. org/10.1002/14651858.CD001027.pub2

R Development Core Team. (2017). R: A language and environment for statistical computing. Retrieved from https://www.r-project.org

Ritterband, L.M., Thorndike, F.P., Ingersoll, K.S., Lord, H.R., GonderFrederick, L., Frederick, C., ... Morin, C.M. (2017). Effect of a webbased cognitive behavior therapy for insomnia intervention with 1-year follow-up: A randomized clinical trial. JAMA Psychiatry, 74(1), 68-75. http://doi.org/10.1001/jamapsychiatry.2016.3249

Seminowicz, D.A., Shpaner, M., Keaser, M.L., Krauthamer, G.M., Mantegna, J., Dumas, J.A., ... Naylor, M.R. (2013). Cognitivebehavioral therapy increases prefrontal cortex gray matter in patients with chronic pain. The Journal of Pain: Official Journal of the American Pain Society, 14(12), 1573-84. http://doi.org/10.1016/j. jpain.2013.07.020

Smith, E.M.L., Bridges, C.M., Kanzawa, G., Knoerl, R., Kelly, J.P., Berezovsky, A., \& Woo, C. (2014). Cancer treatment-related neuropathic pain syndromes-Epidemiology and treatment: An update. Current Pain and Headache Reports, 18(11), 459. http://doi. org/10.1007/s11916-014-0459-7

Smith, E.M.L., Cohen, J.A., Pett, M.A., \& Beck, S.L. (2011). The validity of neuropathy and neuropathic pain measures in patients with cancer receiving taxanes and platinums. Oncology Nursing Forum, 38(2), 133-142.

Smith, E.M.L., Pang, H., Ye, C., Cirrincione, C., Fleishman, S., Paskett, E.D., ... Alliance for Clinical Trials in Oncology. (2015). Predictors of duloxetine response in patients with oxaliplatininduced painful chemotherapy-induced peripheral neuropathy (CIPN): A secondary analysis of randomised controlled trial CALGB/alliance 170601. European Journal of Cancer Care. http:// doi.org/10.1111/ecc.12421

Smith, E.M., Pang, H., Cirrincione, C., Fleishman, S., Paskett, E.D., Ahles, T., ... Oncology, A. for C. T. in. (2013). Effect of duloxetine on pain, function, and quality of life among patients with chemotherapy-induced painful peripheral neuropathy: A randomized clinical trial. JAMA, 309(13), 1359-1367. http://doi. org/10.1001/jama.2013.2813

Tingley, D., Yamamoto, H.T., Kentaro, M., Princeton, H., Keele, L., State, P., \& Imai, K. (2014). Mediation: R Package for Causal Mediation Analysis. Journal of Statistical Software.

Turner, J.A., Holtzman, S., \& Mancl, L. (2007). Mediators, moderators, and predictors of therapeutic change in cognitive-behavioral therapy for chronic pain. Pain, 127(3), 276-86. http://doi. org/10.1016/j.pain.2006.09.005

Yu, L., Buysse, D.J., Germain, A., Moul, D.E., Stover, A., Dodds, N.E., ... Pilkonis, P.A. (2012). Development of short forms from the PROMISTM Sleep Disturbance and Sleep-Related Impairment Item Banks. Behavioral Sleep Medicine, 10(1), 6-24. http://doi.org/10 $.1080 / 15402002.2012 .636266$

Zachariae, R., Lyby, M.S., Ritterband, L.M., \& O’Toole, M.S. (2016). Efficacy of internet-delivered cognitive-behavioral therapy for insomnia-A systematic review and meta-analysis of randomized controlled trials. Sleep Medicine Reviews, 30, 1-10. http://doi. org/10.1016/j.smrv.2015.10.004

Zhuo, M. (2016). Neural mechanisms underlying anxiety—Chronic pain interactions. Trends in Neurosciences, 39(3), 136-45. http://doi. org/10.1016/j.tins.2016.01.006 